\title{
Practical High-Fidelity Frequency-Domain Force and Location Identification
}

\author{
Pooya Ghaderi ${ }^{\mathrm{a}}$, Andrew J. Dick ${ }^{\mathrm{a}, *}$, Jason R. Foley $^{\mathrm{b}}$, Gregory Falbo ${ }^{\mathrm{c}}$ \\ ${ }^{a}$ Rice University, Department of Mechanical Engineering, Houston, Texas, USA \\ ${ }^{b}$ Fuzes Branch, Munitions Directorate, Air Force Research Laboratory, Eglin Air Force \\ Base, Florida, USA \\ ${ }^{c}$ LMS International, A Siemens Business, Troy, Michigan, USA
}

\begin{abstract}
Identifying the force information and location of an impact event is important for predicting and/or monitoring potential damage to the structures. Directly measuring the impact event and/or locating the impact force is not always possible due to the nature of the impact or the structure. In this work, a new force and location identification method is introduced which utilizes a spectral finite element method (SFEM) model of the structure. The identification technique is demonstrated and studied through its application to beam structures in order to identify impulsive loads. Wave propagation data collected with accelerometers placed on the structure are used in order to determine the impact information. When the impact force is applied between the accelerometers, the calculated force is distributed over the two accelerometer positions on either side of the impact location. The location identification process uses the distribution of the identified force information in order to locate the impact position. This method is performed by matching simulated data to the identified force data by tuning the impact location within the numerical model. When a sufficient level of agreement is achieved, the impact location is determined. In order to validate the results of the numerical studies, identified impact forces and locations are calculated for experimental data and good agreement is observed with the
\end{abstract}

\footnotetext{
* Corresponding author

Email address: andrew.j.dick@rice.edu (Andrew J. Dick)
}

Preprint submitted to Computers \& Structures

June 24, 2015 
measured force information and impact locations.

Keywords: Force identification, force localization, impact response, spectral finite element method

\section{Introduction}

Indirect methods for impact force identification have attracted researchers due to the nonlinearity of the impact problem and complexity of impact incidents [1, 2, 3, 4]. Numerous techniques have been developed which uses inverse 5 methods for impact force identification. Some common techniques for force identification are the deconvolution method [1, 2], state variable formulation [5], the sum of weighted accelerations [6], statistical energy analysis method [37], mode superposition technique [38], pseudo-inverse method [39] and the spectral finite element method [7]. The most popular method is the deconvolution technique which uses the assumption of a linear response in order to allow for the application of the convolution integral to identify the force information. For linear systems, the convolution integral of a system's impulse response and the applied force results in the response of the system. This technique has been applied in both the time-domain [1] and the frequency-domain [8].

In a study by Doyle, a time-domain deconvolution technique was successfully developed in order to experimentally obtain dynamic contact laws [1]. Response behavior was monitored by using strain gages affixed to a beam structure and the impulsive load was applied by using a pendulous ball. In other work, Chang and Sun calculated the applied impact force by using an experimental Green's 20 function and time-domain signal deconvolution [9]. The reconstructed force was identified independent of the location of the sensors on a composite beam structure. In both cases, the position of the impact was assumed to be known.

The deconvolution technique is also easily implemented in the frequencydomain for conducting force identification due to the ease of performing the deconvolution calculation since the convolution integral in the time-domain corresponds to a simple multiplication operation in the frequency-domain. In other 
work of Doyle, a frequency-domain deconvolution method was developed and used to experimentally determine contact laws [8]. The impact force was estimated using analytical relationship between contact force and the strain response of structure. In order to improve the quality of the reconstructed force, signal processing techniques were applied on the strain response to avoiding the wrap-around problem [10, 11, 12].

By implementing the finite element method in the frequency-domain, the spectral finite element method (SFEM) was developed [13]. Linear SFEM is highly effective for modeling mechanical wave propagation in simple structures and it has been used for structural impact analysis [14]. As stated by Doyle, "spectrally formulated elements exactly describe the wave propagation dynamics and (in contrast to the conventional element) this means that an element can span all the way from one joint to another without losing fidelity" [14]. SFEM 40 has been used in structural mechanics for applications such as force detection [7] and damage detection [15, 16]. In the work of Doyle and Farris, a DFT-based SFEM was used to study mechanical wave propagation in beams [17]. Ajith and Gopalakrishnan studied wave propagation in plate structures which were stiffened with beam elements by using the spectral finite element method [18].

45 In the work of Mitra and Gopalakrishnan, the discrete wavelet transform was used to prepare a wavelet-domain implementation of SFEM [7]. In their work, the accuracy and computational costs of the wavelet-domain SFEM were determined to outperform an equivalent frequency-domain SFEM when conducting force identification in beams. However, applying the wavelet transform to the system model requires rigorous mathematics while the frequency-domain implementation is more straightforward and intuitive.

Similar to the force identification methods, indirect methods of identification of the impact location have been studied extensively due to limitations in the direct identification of the impact location [1, 8, 19, 20, 21]. Methods for the 55 localization of impacts can be classified into two main categories. The first type of method uses wave propagation speed and arrival time to determine impact location [22, 23, 24, 25, 26]. Methods in the first category take advantage of the 
wave speed and the recorded time for the traveling waves to reach the sensors in order to calculate the position of the impact force. Gaul and Hurlebaus developed a method based on the arrival times of waves at various frequencies [22]. The location of the impact was identified by solving a nonlinear equation extracted from the speed of the traveling waves. The second type of localization of impact force is performed by minimizing an error function which is defined based on the impact force and the system response [27, 28, 29]. The system response can be obtained from a system model or transfer function which is derived analytically, numerically, or experimentally [30].

Additionally, it is possible for the two methods to be used simultaneously to accurately locate the impact force. In the recent work of Liang et al., elements of the two methods were combined to create a distributed coordination algorithm for locating the impact force with greater computational efficiency [31]. However, further studies are required in order to fully explore the effectiveness of this approach.

In this study, a method is presented for identifying and locating impulsive forces by using a frequency-domain SFEM-based force identification and localization method. This method is implemented and studied by using a beam structure. By assuming linear behavior for the structure, the impact force is reconstructed by using a SFEM model. With the new approach that is introduced in this paper, the impact force is identified independent of the impact position, which improves the accuracy of the results and reduces computational costs. After identifying the impact force, the obtained force information is used to locate the impact force on the structure iteratively. Since the impact force is already calculated, only a few iterations is required in order to locate the impact which saves computational time comparing to previous methods that identify and locate the impact force simultaneously. Some of this material has 85 previously been presented by the authors at conferences and can be found in the corresponding proceedings [32, 33]. In this work, a combined impact force identification and location identification method with necessary signal processing techniques that improves the quality and accuracy of the results is presented. 


\section{Background}

The force identification method proposed in this paper uses the DFT-based spectral finite element method [14]. In this study, the method is applied and studied by using a free-free beam structure. The nodes in the model correspond to the positions of the accelerometers on the beam structure. An Euler-Bernoulli beam model is used in this study to represent the beam structure since shear deformations are negligible and impact forces have relatively low frequency content with respect to the frequency range that Euler-Bernoulli beam model can accurately represent wave propagation [14]. While the SFEM-based method can be applied to response data with much higher frequency content, the impact force used in this study is selected because it can easily be realized experimentally. For impacts with higher frequency content, higher order models such as Timoshenko beam model should be used. The SFEM model for the Euler-Bernoulli beam uses the analytical solution of the beam equation to form the shape functions and to prepare the dynamic stiffness matrix. The frequency-domain deflection and slope of the beam are defined in Eqs. (1) and (2), respectively.

$$
\begin{aligned}
\hat{v}(x, \omega) & =\hat{A} e^{-i \hat{\beta} x}+\hat{B} e^{-\hat{\beta} x}+\hat{C} e^{i \hat{\beta} x}+\hat{D} e^{\hat{\beta} x}, \\
\hat{\phi}(x, \omega) & =\hat{\beta}\left(-i \hat{A} e^{-i \hat{\beta} x}-\hat{B} e^{-\hat{\beta} x}+i \hat{C} e^{i \hat{\beta} x}+\hat{D} e^{\hat{\beta} x}\right), \\
\hat{\beta} & =\sqrt[4]{\left(\left(\omega^{2} \rho A-i \omega \eta A\right) / E I\right)},
\end{aligned}
$$

where beam deflection, slope, and the spatial coordinate are represented by $\hat{v}$, $\hat{\phi}$, and $x$, respectively. The hat symbol $\left({ }^{\wedge}\right)$ denotes frequency-domain variables and each variable is a function of frequency. The characteristic root of the equation of motion is represented by $\hat{\beta}$ which is defined in Eq. (33), where $\omega(1 / s)$ represents frequency and $E I\left(\frac{k g \cdot m^{3}}{s^{2}}\right), \rho A\left(\frac{k g}{m}\right)$, and $\eta A\left(\frac{k g}{m . s}\right)$ correspond to the flexural rigidity, mass per length, and viscous damping coefficient from the Euler-Bernoulli beam model, respectively. The parameters $\hat{A}, \hat{B}, \hat{C}$, and $\hat{D}$ are frequency-domain deflection coefficients. The boundary condition at the spatial coordinates of $x=0$ and $x=L$ for a beam element of length $L$ are applied to the deflection function and the relationship between the boundary values and 
the deflection coefficients is written in matrix form, as shown in Eq. (4).

$$
\left\{\begin{array}{l}
\hat{v}_{1} \\
\hat{\phi}_{1} \\
\hat{v}_{2} \\
\hat{\phi}_{2}
\end{array}\right\}=\left[\begin{array}{cccc}
1 & 1 & 1 & 1 \\
-i \hat{\beta} & -\hat{\beta} & i \hat{\beta} & \hat{\beta} \\
e^{-i \hat{\beta} L} & e^{-\hat{\beta} L} & e^{i \hat{\beta} L} & e^{\hat{\beta} L} \\
-i \hat{\beta} e^{-i \hat{\beta} L} & -\hat{\beta} e^{-\hat{\beta} L} & i \hat{\beta} e^{i \hat{\beta} L} & \hat{\beta} e^{\hat{\beta} L}
\end{array}\right]\left[\begin{array}{c}
\hat{A} \\
\hat{B} \\
\hat{C} \\
\hat{D}
\end{array}\right]
$$

where $\hat{v}_{i}$ and $\hat{\phi}_{i}$ for $i=1,2$ are the beam element's nodal deflection and slope functions, respectively. The subscripts 1 and 2 denote the values of the functions of the nodes at $x=0$ and $x=L$, respectively. For simplicity, the vectors and matrix in Eq. (4) are redefined in Eq. (5) where the deflection and coefficient vectors are represented by $\{\hat{u}\}$ and $\{\hat{R}\}$, respectively. The matrix that relates the two is defined by $[\hat{Q}]$. The deflection coefficients are obtained by inverting the matrix in Eq. (5) and pre-multiplying both sides of the equation by the matrix inverse, as shown in Eq. (6). The inverted matrix in Eq. (6) is redefined as $[\hat{G}]$ for simplicity.

$$
\begin{aligned}
\{\hat{u}\} & =[\hat{Q}]\{\hat{R}\}, \\
\{\hat{R}\} & =[\hat{Q}]^{-1}\{\hat{u}\}=[\hat{G}]\{\hat{u}\}
\end{aligned}
$$

The beam deflection is expanded in terms of the deflections and slopes at $x=0$ and $x=L$ as defined in Eq. (7) where $\hat{g}_{j}(x), j=1,2,3,4$ represents the shape functions of the beam element. The shape functions of the beam element, defined in Eq. (8), are written in terms of the elements of matrix $[\hat{G}]$ and exponential functions of $\hat{\beta}$.

$$
\begin{aligned}
\hat{v}(x, \omega) & =\hat{g}_{1}(x, \omega) \hat{v}_{1}+\hat{g}_{2}(x, \omega) \hat{\phi}_{1}+\hat{g}_{3}(x, \omega) \hat{v}_{2}+\hat{g}_{4}(x, \omega) \hat{\phi}_{2}, \\
\hat{g}_{j}(x, \omega) & =\hat{G}_{1 j} e^{-i \hat{\beta} x}+\hat{G}_{2 j} e^{-\hat{\beta} x}+\hat{G}_{3 j} e^{i \hat{\beta} x}+\hat{G}_{4 j} e^{\hat{\beta} x}, \quad j=1,2,3,4 .
\end{aligned}
$$

The dynamic stiffness matrix $[\hat{K}]$ of the beam element relates the moments $\hat{M}$ and shear forces $\hat{V}$ at the nodes of the beam element to the deflections and slopes of the beam elements at the corresponding nodes, as shown in Eq. (9). The dynamic stiffness matrix is defined in terms of the derivatives of the shapes 
functions, the structure's geometry, and the material properties.

$$
\left[\begin{array}{c}
\hat{M}_{1} \\
\hat{V}_{1} \\
\hat{M}_{2} \\
\hat{V}_{2}
\end{array}\right]=E I\left[\begin{array}{cccc}
-\hat{g}_{1}^{\prime \prime}(0) & -\hat{g}_{2}^{\prime \prime}(0) & -\hat{g}_{3}^{\prime \prime}(0) & -\hat{g}_{4}^{\prime \prime}(0) \\
-\hat{g}_{1}^{\prime \prime \prime}(0) & -\hat{g}_{2}^{\prime \prime \prime}(0) & -\hat{g}_{3}^{\prime \prime \prime}(0) & -\hat{g}_{4}^{\prime \prime \prime}(0) \\
\hat{g}_{1}^{\prime \prime}(L) & \hat{g}_{2}^{\prime \prime}(L) & \hat{g}_{3}^{\prime \prime}(L) & \hat{g}_{4}^{\prime \prime}(L) \\
\hat{g}_{1}^{\prime \prime \prime}(L) & \hat{g}_{2}^{\prime \prime \prime}(L) & \hat{g}_{3}^{\prime \prime \prime}(L) & \hat{g}_{4}^{\prime \prime \prime}(L)
\end{array}\right]\left\{\begin{array}{c}
\hat{v}_{1} \\
\hat{\phi}_{1} \\
\hat{v}_{2} \\
\hat{\phi}_{2}
\end{array}\right\} .
$$
accelerometers located near the location of the impact. Although the exact lo- 


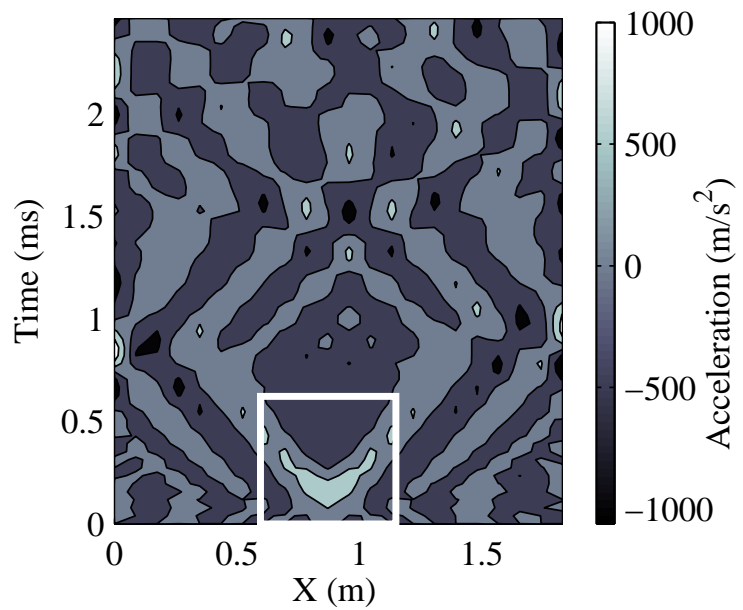

Figure 1: Simulated wave propagation (acceleration data) in a free-free beam. The boxed area corresponds to data which is selected for analysis. This area corresponds to only a section of the structure and does not include reflections.

cation of the impact is unknown, it is assumed that the general area in which the impact occurs is known.

The simulation acceleration data of wave propagation through the beam structure is illustrated in Fig. 1. The figure illustrates the variation of acceleration at each location of the beam through time. This response is used in order to identify the impact force. After the impact occurs, mechanical waves travel through the beam structure and reflect when they reach the ends. The reflections and dispersion in beam structures increase the difficulty of performing successful force identification, especially if it is not possible to mount sensors at the ends of the structure. In this force identification method, the impact force is calculated by using a SFEM model and a subset of the response data. This subset of the displacement and slope data corresponds to a reduction in both the spatial and temporal dimensions around the location where the propagated wave originated, as illustrated by the box in Fig. 1. The subset contains the response data collected from a few sensors around impact location. In the time domain, the initial response due to the impact is used and the rest of the response which 


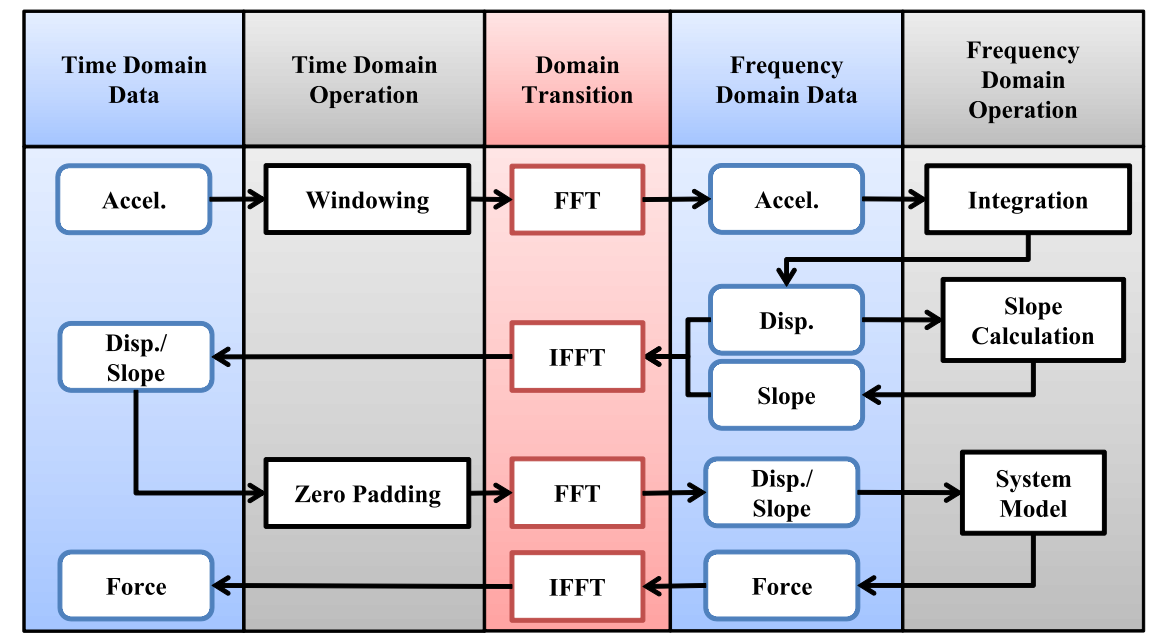

Figure 2: Flowchart illustrating the main steps of the force identification scheme.

is the reflections of the wave is omitted. The resolution of the data is based on the experimental setup. In this study, the equipment and experimental setup is explained in section [? ]. Note that acceleration data is presented in this figure. This subset of data chosen for the identification procedure is selected so that it does not contain reflections. The data subset is used with a beam segment model to calculate the desired force information.

A flowchart of the force identification procedure is presented in Fig. 2 The acceleration data from the structure is transformed into the frequency-domain by using the fast Fourier transform (FFT) and is integrated twice to obtain displacement. The slope information required for the identification process is calculated by using a shape function-based method. Zero-padding is applied to the response data in order to address the FFT's assumption of periodicity. The dynamic stiffness matrix of the SFEM model for a segment of the beam is used to calculate the force information in the frequency-domain. Detailed descriptions of each step are presented in the following subsections. 


\subsection{Time-Domain Signal Conditioning}

190

placement data right after the impact which is used for the force identification problem is negligibly influenced by the drift. 


\subsection{Slope Calculation}

The acceleration data only provides information regarding the one-dimensional

220

force identification procedure to a beam structure requires both deflection and slope information. Therefore, it is necessary to calculate the corresponding slope response at each location. This is accomplished by taking advantage of the kinematics of the beam structure. In the slope calculation process, the slope using deflection data at each node. The deflection coefficients, $\hat{A}, \hat{B}, \hat{C}$, and $\hat{D}$, of each four-node beam element are calculated by substituting the deflection values at the four nodes for the specified range of frequencies into the deflection equation and solving for the deflection coefficients. Slope information is obtained

\subsection{SFEM Model for Force Identification}

The SFEM model used in the force identification process represents a segment of the beam structure. The implementation of the force identification method presented here utilizes the response data from the five accelerometer positions in the vicinity of the impact force. The spectral finite element model is constructed by using a throw-off element at each end of the segment. For these conditions, the frequency-domain dynamic stiffness matrix is prepared to relate the displacement and slope information to the corresponding force and moment information for the beam segment. Although this model differs considerably 245 from the full structure model, a response predicted by the segment model with the same impact force previously considered, shown in Fig. 3, matches perfectly 


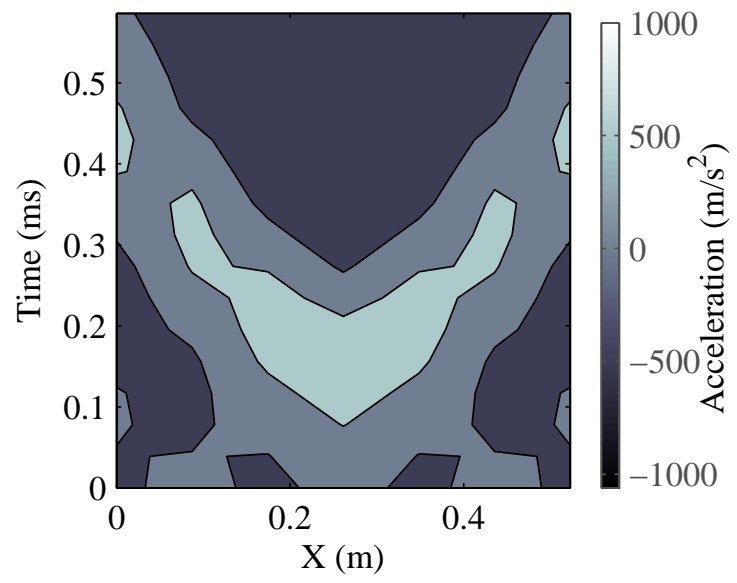

Figure 3: Simulated mechanical wave propagation (acceleration data) using segment model which includes two throw-off elements.

with the local response behavior of the full structure, identified by the box in Fig. 1

Due to the nature of SFEM, this force identification method calculates nodal forces. For conditions where the location of the applied load and the accelerometer are collocated, the force identification process does not require any additional steps in order to obtain the identified force information. However, for cases where the impact is applied between two accelerometers, point forces are calculated at the neighboring nodes when the force identification procedure is applied. The summation of the two force time histories produces the identified force when certain conditions are satisfied. These conditions and the justification for this process is discussed in section 4 where the location identification process is presented. This approach allows for the impact force information to be efficiently identified independent of the exact location of the impact.

\subsection{Numerical Implementation and Parametric Study}

The force identification procedure is first applied to the deflection and slope data from the numerical simulations. This eliminates the potential error introduced by the deflection and slope calculations and allows for the study to 
focus on the influence of other conditions on the performance of the force identime-domain methods, have been found to display limitations with regard to high frequency performance [36]. For this reason, experimental response data is also used in order to validate the performance of the force identification method.

For the ideal conditions, the point force is detected at the location of the 


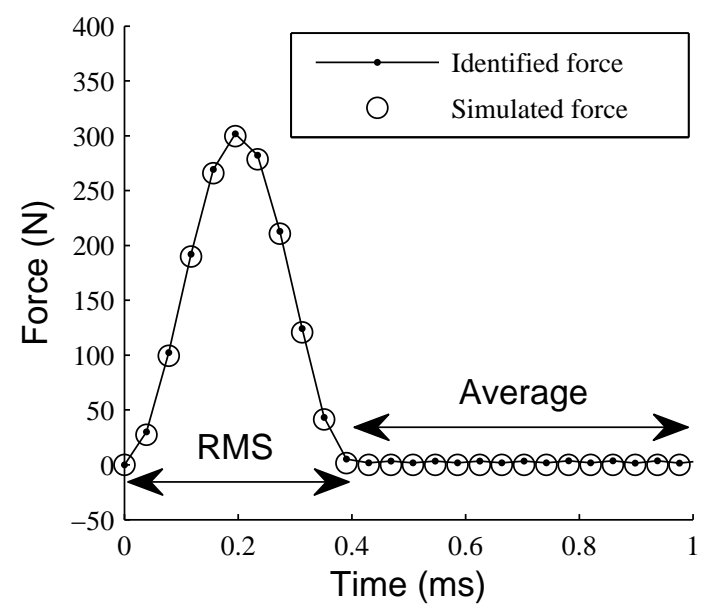

Figure 4: Force information calculated for the simulated response for an $18 \mathrm{ft}(5.49 \mathrm{~m})$ long beam. For this nominal case, there is strong agreement between the identified (connected points) and simulated (circles) force information.

impact, which is at node 33 at a position of $8.85 \mathrm{ft}(2.70 \mathrm{~m})$ from the reference end of the beam. The peak calculated forces at the other nodes within the subset of data analyzed have values less than $6 \mathrm{~N}(<2 \%)$, which are easily distinguished from the identified impact force. The force information identified from the simulated response, shown in Fig. 4, agrees very well with the simulated force. In order to quantify the accuracy of the force identification method, a Root Mean Square (RMS) error is calculated by comparing the identified force values to the values used in the simulation. The RMS error for impact force identification [2] provides quantitative information about the average error through the impact time history. The quantification of the error is performed by first separating 305 the time series into two regions: the impulsive load and the remaining portion of the time history, as shown in Fig. 4. For the nominal case, the RMS error is $2.72 \mathrm{~N}$ during the impact and an average value of $2.46 \mathrm{~N}$ exists for the impact force considered over the remainder of the $1 \mathrm{~ms}$ presented. These errors are quite small when compared with the $300 \mathrm{~N}$ peak value of the impulsive load, less than 1 percent. The presence of error in the identified force information for 

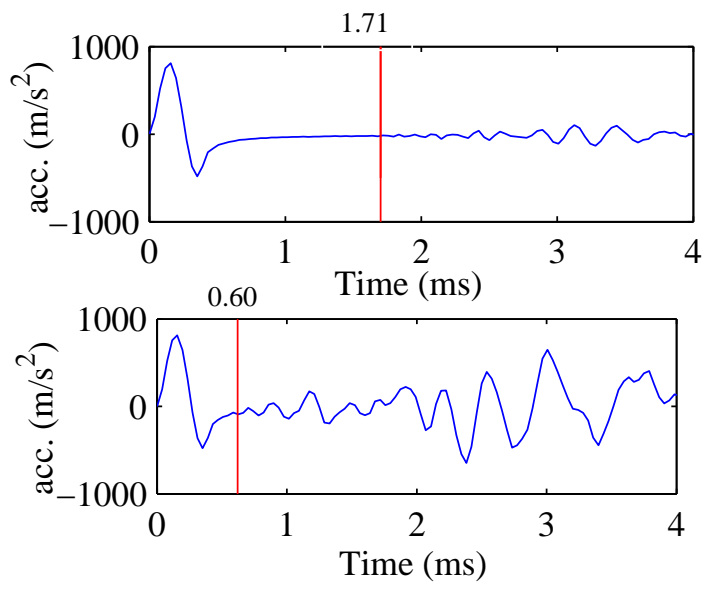

Figure 5: Acceleration response at a node near the center of the beam for (top) $18 \mathrm{ft}(5.49 \mathrm{~m})$ beam and (bottom) $6 \mathrm{ft}(1.83 \mathrm{~m})$ beam. Vertical lines identify when the reflection returns to the impact location.

the nominal case results from the use of the segment model and how it differs from the complete structure. However, the use of the segment model provides the means for the practical implementation of the force identification method.

The geometric and loading properties corresponding to the results presented in Fig. (4provide nominal conditions for performing force characterization. Variations of these properties are studied in order to determine how they influence the ability of the force identification method to accurately calculate the applied impulsive load. This includes the effects of (i) varying the length of the structure, (ii) using calculated slope information instead of simulated slope information, (iii) varying the location of the applied force, (iv) varying the duration of the impulsive load, and (v) varying the length of the response data analyzed. During each part of the parametric study, the values of the parameters not currently being investigated are set to the values corresponding to the ideal conditions. The acceleration signals used in the numerical studies do not contain noise.

\section{(i) Structure Length}

In order to study the influence of the length of the structure on the accuracy 
of the calculated force information, the force identification process is applied to response behavior simulated for a range of lengths from $0.5 \mathrm{ft}(0.15 \mathrm{~m})$ to $18 \mathrm{ft}$ $(5.49 \mathrm{~m})$. In each case, the impact force is applied at a node in the middle of the structure. While the length of the structure is varied, all other conditions are maintained and the simulated displacement and slope information is used for the force calculation. The main effect of varying the length of the structure is to change the length of time before the reflected waves return the location where the impact force was applied. This effect is illustrated in Fig. 5 ,

The vertical lines in Fig. 5 indicate the time when the reflection is observed in the acceleration data. For the nominal case with an $18 \mathrm{ft}(5.49 \mathrm{~m})$ long beam, the reflection is observed after $1.71 \mathrm{~ms}$. When the beam length is reduced to $6 \mathrm{ft}(1.83 \mathrm{~m})$, reflections are observed after only $0.60 \mathrm{~ms}$. Since the shorter length is one-third the initial length, it takes roughly $1 / 3$ of the time before the 340 reflected wave returns. This time depends on the length of the beam as well as the wave speed. Since wave propagation is dispersive in beam structures, the components of the mechanical waves at different frequencies travel with different speeds. The return of the reflected waves is identified by detecting oscillations in the response data after the initial wave.

Error information is obtained by applying the force identification scheme to the response data simulated for beam structures of lengths within identified range. The RMS error values (*) obtained by comparing the simulated and identified force values during the impulsive force and the average identified force values (o) after the impulsive force are presented in Fig. 6 Examination of these error values reveal that as the length of the structure is decreased, the values of both error measures appear to increase along a logarithmic profile. This is confirmed by using a least squares approach to fit the RMS error values to a logarithmic curve: Error $=-7.55 \ln (L)+14.67$ with $R^{2}=0.9999$. A similar logarithmic curve is also found to fit the trend of the values of the other error 355 measure (not shown). In addition to displaying the same logarithmic behavior, it can also be seen in Fig. 6 that the values of the two error measures are very close at all of the lengths considered. This suggests that the influence of 


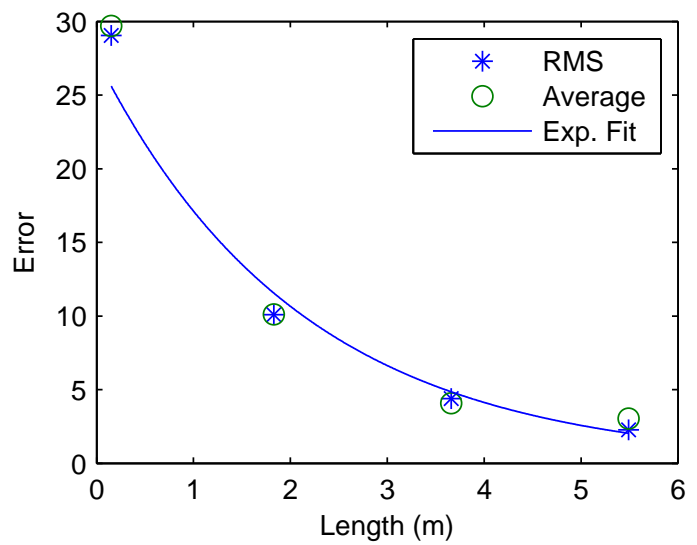

Figure 6: Force identification error with respect to the length of the structure. RMS error $\left(^{*}\right)$ during the impulsive load, average force (o) after the impulsive load, and a logarithmic curve (-) fit to the RMS error values are presented.

structure length on the performance of the force identification method is not significantly affected by the magnitude of the force information. The increase in the error measure values which are observed for beam structures with shorter lengths is the result of the shorter amount of time before the reflected wave returns to the position on the structure where the force was applied. With less time before the reflections occur, the subset of data which can be used by the force identification method is reduced and greater deviation exists between the data and the behavior expected by the segment model.

\section{(ii) Slope Calculation}

The slope calculation method is tested by using deflection and slope data from numerical simulations. The slope information calculated from the simulated deflection data demonstrates excellent agreement with the simulated slope information. The maximum difference between the calculated and simulated slope data is between nine and ten orders of magnitude less than the values of the slope from the simulation.

The error introduced by including the slope calculation in the force identification procedure is determined to be negligible for the simulated response 
data. By using the shape function method to calculate the slope information, the error in the identified impulsive force does not change from the nominal case. However, noise in the response can cause the accuracy of the calculated slopes, and subsequently the identified force information, to be reduced. The calculated slope values and the corresponding identified force information are predicted to be accurate as long as the signal-to-noise ratio remains greater than about $100: 1$. This limitation is addressed in the experimental verification by using accelerometers with sufficiently high sensitivity.

\section{(iii) Impact Location}

All of the calculated force information previously presented corresponds to impulsive loading near the center of the structure. In order to investigate the influence of the impact location on the accuracy of the calculated force information, the impact force is applied at a number of locations on the structure. The impact positions considered range from $1.43 \mathrm{ft}(0.44 \mathrm{~m})$ to $9.14 \mathrm{ft}(2.79 \mathrm{~m})$ from the end of the $18 \mathrm{ft}(5.49 \mathrm{~m})$ long structure. As a result of moving the impact location closer to the end of the structure, the reflections return to the impact location more quickly. The increase in error resulting from moving the impact location closer to the end of the structure is illustrated in Fig. 7.

The RMS error from the identified impulsive load and the average identified force values after the impact both increase as the impact position moves closer to the end of the structure. Because moving the impact location has the same effect as changing the length of the structure, i.e. reducing the length of time before the propagating wave returns to the impact location, the error values display the same logarithmic trend. In this case, the logarithmic curve fit to the RMS error values takes the form Error $=-4.636 \operatorname{Ln}\left(x_{\text {impact }}\right)+6.6867$ with $R^{2}=0.9916$. 400 When accounting for the fact that when the impact load is applied in the middle of the structure, the length over which the wave propagates before reflecting is half of the length of the structure, the data in Fig. 6 and Fig. 7 are very close to each other. While all of the conditions studied correspond to the same geometry and material properties, these factors influence the wave propagation speed and subsequently the length of time required for the propagating wave to be reflected 


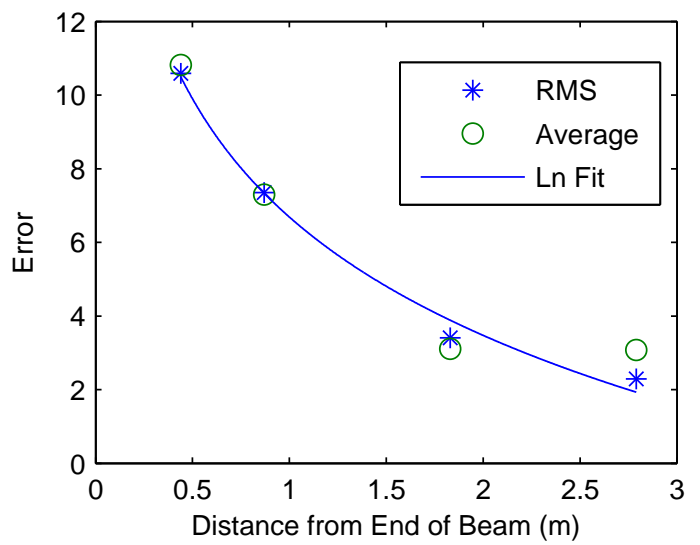

Figure 7: Force identification error with respect to the length of the structure. RMS error $\left(^{*}\right)$ during the impulsive load, average force (o) after the impulsive load, and a logarithmic curve (-) fit to the RMS error values are presented.

and return to the impact location.

\section{(iv) Impact Duration}

Next, the influence of impact duration on the performance of the force identification method is studied. System responses are simulated for impulsive loads with half and twice the original duration. Changing the impact duration affects the response of the system in two significant ways. The first and most straightforward effect of changing the impact duration is that the amount of time between the end of the impulsive load and when the reflection returns the impact position is increased (reduced duration) or decreased (extended duration). The effect on the performance of the force identification method will be similar to changing the distance between the impact position and the end of the structure. The second effect of changing the impact duration is that shorter/longer duration loads will contain a larger/smaller range of frequency content. Since the force identification method utilizes the SFEM, is it able to accommodate the higher frequency content and the change in the error results from the travel time of the propagating and then reflected wave. The two error measures for these conditions and the nominal case are presented in Fig. 8 


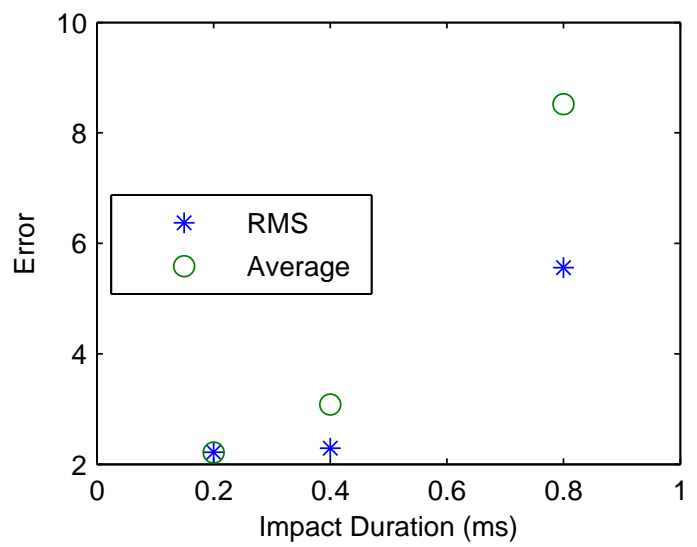

Figure 8: Force identification error with respect to the impulsive load duration. RMS error (*) during the impulsive load and average force (o) after the impulsive load are presented.

From the data presented in Fig. 8 it can be seen that for a shorter impact duration, the RMS error remains the same and the average force after the impact is slightly decreased. When the impact duration is increased to $0.8 \mathrm{~ms}$, both error measures increase in value. The average force after the impact increases more the RMS error during the impact. However, these variations of the impact duration result in smaller increases in the error measures than the other parameters.

\section{(v) Length of Response Data}

The influence of the length of the response data on the accuracy of the reconstructed force is next studied. When less of the response data is available, the wave propagation data may not have fully decayed. This will require the application of more aggressive exponential windowing in order to reduce the response to zero by the end of the data set. When this is done, the influence of the exponential windowing on the data at the beginning of the data set which is used for the force identification process is more significantly affected. This can increase the error in the identified force information. Properties such as damping and the boundary conditions of the structure can influence the length of time required for the propagating wave to decay and subsequently the length 


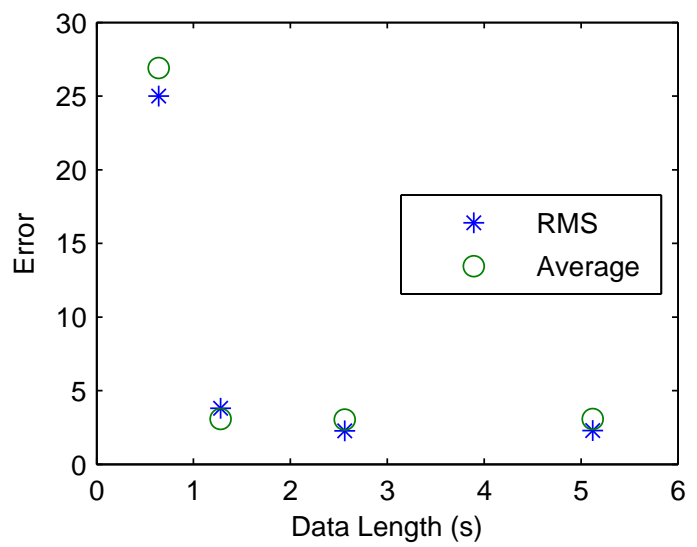

Figure 9: Force identification error with respect to the length of the response data. RMS error (*) during the impulsive load and average force (o) after the impulsive load are presented.

of the data set required to produce accurate results. In this study, these properties are kept constant and various lengths of response data are used to calculate the applied force. The error measures for the identified force information when using response data sets of lengths ranging from $5.12 \mathrm{~s}$ to down to $0.64 \mathrm{~s}$ are presented in Fig. 9 .

In this figure, it can be seen that above a threshold which lies between $0.64 \mathrm{~s}$ and $1.28 \mathrm{~s}$, the error measures for the identified force information is close to the values for ideal conditions. However, after the length of the response data set is decreased to $0.64 \mathrm{~s}$, both of the error measures increase by an order of magnitude. These results suggest that for a given set of conditions, a certain amount of time is required for the response of the structure to sufficiently decay in order for the force identification scheme to provide accurate results. Additionally, when the response data of length $0.64 \mathrm{~s}$ is used, the identified force information includes a consistent offset between the identified force values and the simulated force values, as shown in Fig. 10. Based upon the results of this numerical study, the presence of this characteristic in identified force information indicates that a longer amount of response data is required to improve the accuracy of the identified force. 


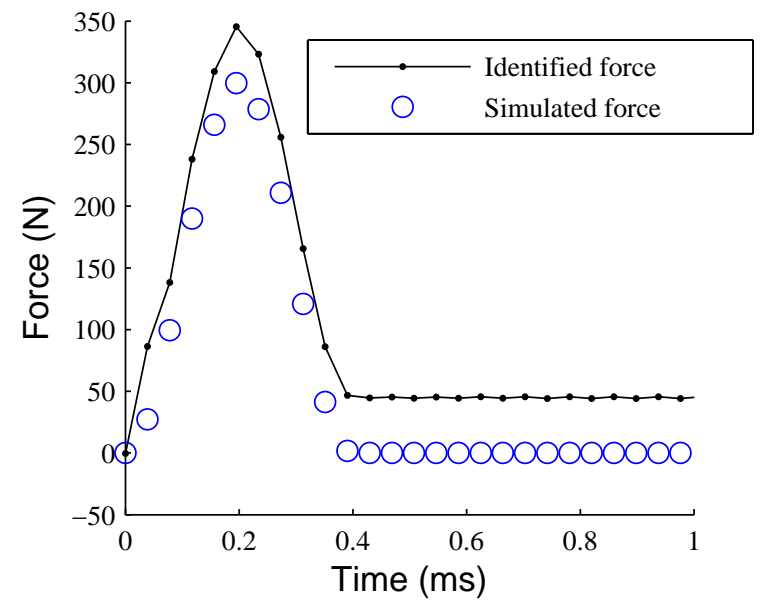

Figure 10: Identified (connected points) and simulated (circles) force information when only one quarter of the nominal length of the data set is used.

Through this parametric study, the influence of (i) structure length, (ii) slope calculation, (iii) impact location, (iv) impact duration, and (v) length of response data on the performance of the force identification method was studied. The accuracy of the reconstructed impact force is qualitatively high comparing to previous works 1, 2, 4]. However, the variation in different case studies and measurement methods is a significant factor in the accuracy of the results. For instance, using calculated slope information in this work is a source of error specific to this work which can be enhanced by using gyro-sensors. Additionally, the focus of this study is on the practicality of the identification method under high frequency content impact with one single measurement, minimum number of sensors and no extensive experimental or numerical modeling. The SFEM model is an accurate model for modeling the high frequency waves. The local measurement is a practical way to use the fewest sensors possible and provides the capability to use the method with any boundary conditions due to the fact that only the initial response is required for the force identification process. Error in the identified force information was determined to increase for decreasing structure length, impacts applied closer to the end of the structure, 
longer impact durations, and when using response data with lengths below a system dependent threshold. The length of the response data was determined to have the potential to affect performance the most of the parameters studied. Changes to the structure length, impact location, and impact duration where determined to all have similar effects on the performance of the force identification method. When a signal-to-noise ratio of greater than 100:1 is maintained, the error introduced by the slope calculation is expected to be negligible.

\section{Impact Location Identification}

The force identification method reconstructs the impact force in terms of nodal forces. If the impact force is applied between the accelerometers, the distribution of the impact force is utilized to locate the impact location. A freebody diagram of the element with the applied impact force $F$ and the nodal forces, $F_{1}$ and $F_{2}$, is shown in Fig. 11] By applying Newton's method, the summation of vertical forces and the summation of moments are calculated and presented in Eq. (10) and Eq. (11), respectively.

$$
\begin{aligned}
\sum F & =F-F_{1}-F_{2}=m \ddot{V}_{c} \\
\sum M_{c g} & =\Delta M-F_{1} \frac{L}{2}+F_{2} \frac{L}{2}+F\left(\frac{L}{2}-x\right)=\frac{m L^{2}}{12} \ddot{\theta}_{c}
\end{aligned}
$$

where the mass of the element, displacement of the center of mass of the element, and the rotation of the element are represented by $m, V_{c}$, and $\theta_{c}$, respectively. The impact force is applied at the distance $x$ from the first node of the beam element of length $L$.

When the element is relatively short and the mass per length $\rho A$ of the beam is small, the mass of the element is relatively small and the inertial force can be considered negligible with respect to the applied force. For instance, the mass of a $3.43 \mathrm{in}(8.71 \mathrm{~cm})$ element of an Aluminum beam with a $1 \mathrm{in}^{2}\left(6.45 \mathrm{~cm}^{2}\right)$ cross section is $0.137 \mathrm{~kg}$ (weighing $1.34 \mathrm{~N}$ ) which is quite small compared to the

$300 \mathrm{~N}$ impact that is applied. By considering the mass of the element negligible, the equation for the summation of vertical forces is simplified to $F_{1}+F_{2} \approx F$. 


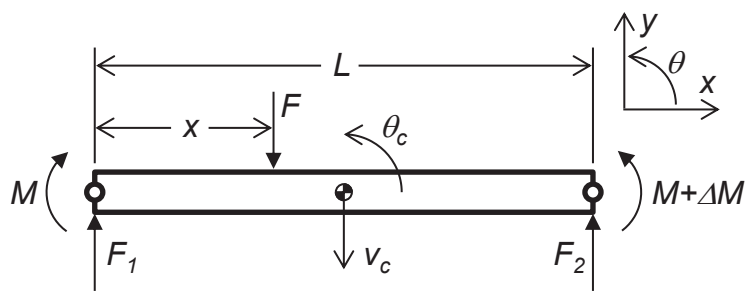

Figure 11: Free-body diagram of the beam element with the impact force $F$ and the nodal forces, $F_{1}$ and $F_{2}$.

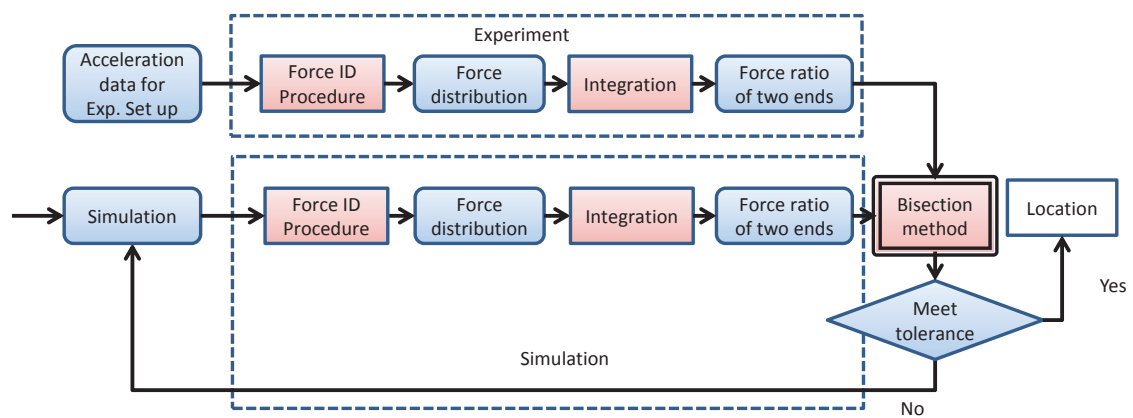

Figure 12: Flowchart of the location identification algorithm.

When these assumptions are satisfied, the summation of the two nodal forces is equal to the applied impact force. As a result, it is possible to determine the impact force without knowledge of the exact location of the impact. This can significantly reduce the computational costs when compared to other approaches [2, 3, 4].

In order to calculate the impact location from the nodal forces, a straightforward nonlinear numerical solver is used. A flowchart illustrating the algorithm used for location identification is presented in Fig. 12. The first step is to determine the distribution of the calculated impact force at the two ends of the element by using the experimental data collected from the accelerometers. The ratio of the impulses at the two neighboring accelerometers is used in this process. Then, simulations are performed with the beam model to produce re- 

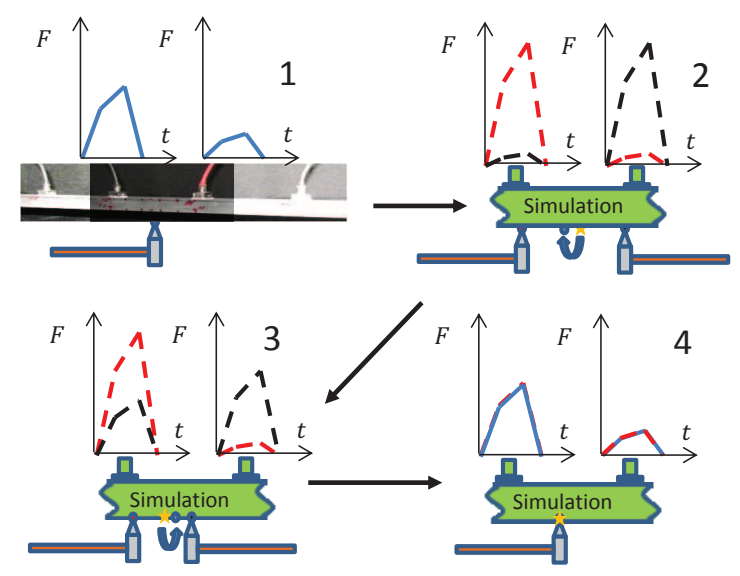

Figure 13: Implementation of the bisection method for the location identification process.

sponse data for comparison. In order for this process to be effective, the model must accurately represent the structure. Known material properties and system geometry are used for the model and the damping parameter value is identified by comparing the simulated response of the model to the response of the experimental system. In the simulation portion of the process illustrated in Fig. 12 an extra node is added between the accelerometer positions for applying the impact force and predicting the response of the structure. However, only data from the nodes which correspond to the accelerometer locations are used in the location identification process. By using the ratio of the impulses for the experimental data and the simulation data, the location of the impact is calculated with the bisection method.

Although the relationship between the impulse ratio and the impact location is nonlinear, it is also a one-to-one relationship. This allows for the use of rootfinding methods such as the bisection method. In order to start this process, two points are chosen close to the two nodes of the element on which the impact force was applied. The distance from the two starting points to the nodes is 530 chosen to be $5 \%$ of the length of the element. This level of precision is selected as it corresponds to a practical limitation identified in the location identification 


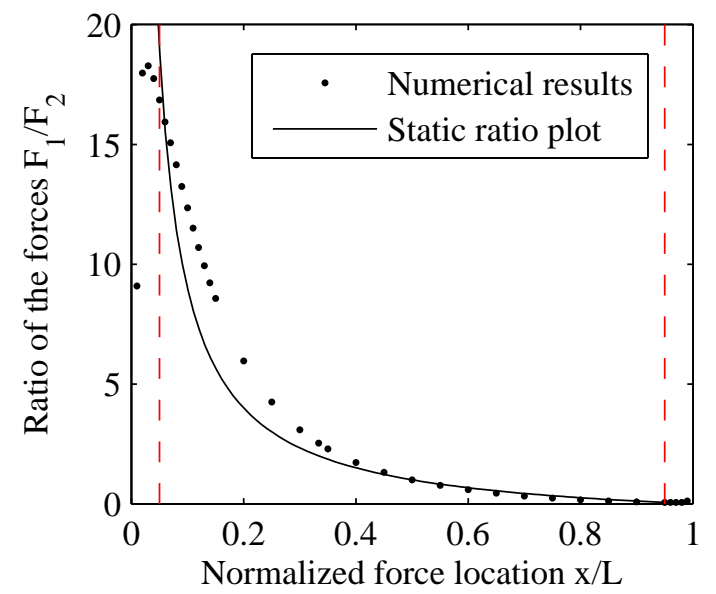

Figure 14: Impulse ratio as a function of impact location for the SFEM model $(\bullet)$ and the analytical model $(-)$. Red dashed lines indicate $\pm 5 \%$ of location between nodes

procedure. The simulated impulse ratios for the two points are then compared to the impulse ratio for the experimental data. Based on the differences between these ratios, one of the initial points is discarded and a simulation is performed to obtain a simulated impulse ratio for a new point directly between the two initial points. The bisection method is continued in this way, as illustrated in Fig. 13, until the difference between the experimental impulse ratio and a simulated impulse ratio is less than the prescribed tolerance. The impact position used for the final simulated case provides the impact location for the experimental 540 data.

The ratio of the identified impact forces at the two nodes adjacent to the impact location, calculated by using the simulation data, is shown in Fig. 14 These results are for a specific case and the details of this relationship are determined to be influenced by material properties, element length, and sampling frequency. In order to study the relationship between the impulse ratio and the location of the impact, the analytical model presented in Fig. 11]is revisited. By using the relationship between the impact force and nodal forces and similarly considering the rotational inertia to be negligible, Eq. (11) is simplified. If the 


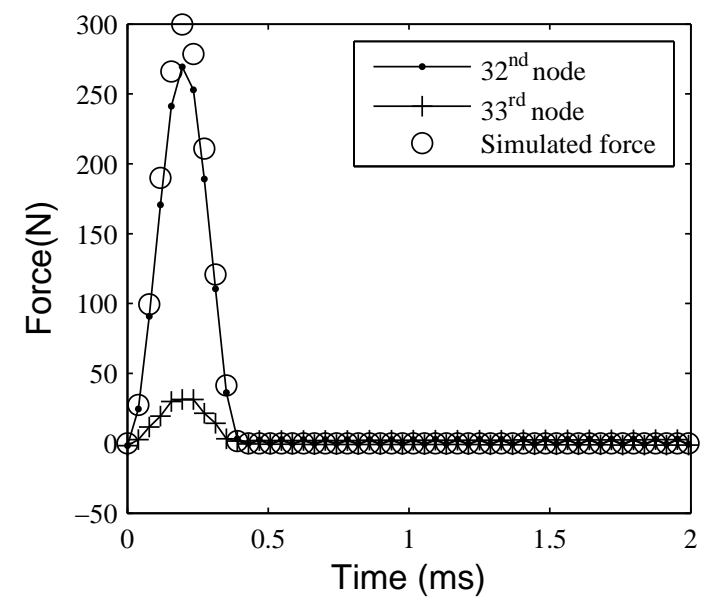

Figure 15: Force information calculated using simulation data. The force is applied at $20 \%$ of the element length from the $32^{\text {nd }}$ node.

deformation of the beam element is sufficiently small and the moment difference $\Delta M$ is considered negligible, an analytical formula for the impact force ratio is obtained. This formula, $F_{1} / F_{2}=(x-L) / x$, provides the approximate ratio between the two nodal forces. The impact force ratio for these assumptions is plotted as a function of position in Fig. 14 along with impulse ratio data from numerical studies performed with the SFEM model of the beam structure. The analytical results qualitatively agree with the numerical results. The slight difference between the two is believed to result from neglecting the deformations of the beam element in the analytical model.

The impact force location identification process is applied to simulation results in order to study the method. Representative results are presented for a case where the impact force is applied between nodes 32 and 33 on the $18 \mathrm{ft}$ $(5.49 \mathrm{~m})$ Aluminum beam at $20 \%$ of the element length $(0.2 L)$ from node 32. The force information calculated from the simulated response data is presented in Fig. 15. As anticipated, larger force values can be seen for the node closer to the impact location. By combining the force information, the identified impact 565 force is calculated. These results, presented in Fig. 16, display strong agreement 


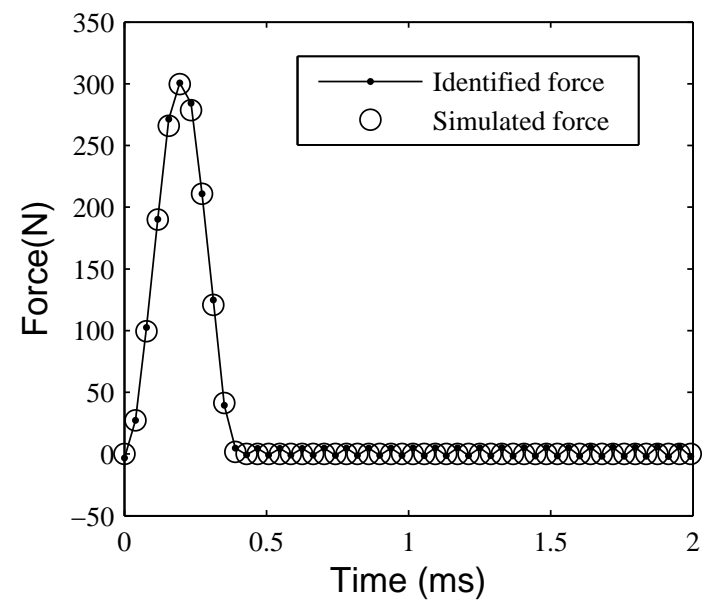

Figure 16: Summation of identified forces calculated using simulation data. The force is applied at $20 \%$ of the element length from the $32^{\text {nd }}$ node.

between the identified force and the simulated force without knowledge of the impact location. The RMS error for the impact and the average for the remainder of the $2 \mathrm{~ms}$ period shown in the figure are $2.98 \mathrm{~N}$ and $2.76 \mathrm{~N}$, respectively. By analyzing the two calculated force curves presented in Fig. 15, an impulse ratio of 8.33 is calculated. The impact location is calculated to be at a distance of $0.19 \mathrm{~L}$ from node 32 . This corresponds to an error of $1 \%$ of the element length or 0.034 in $(0.09 \mathrm{~cm})$ on the $18 \mathrm{ft}(5.49 \mathrm{~m})$ beam structure.

\section{Experimental Verification}

The performance of the force identification method is further studied with experimental response data in order to verify the performance observed by using simulated response data. The experimental setup is a $6 \mathrm{ft}(1.83 \mathrm{~m})$ long aluminum bar with a square cross-section and width of 1 in $(25.4 \mathrm{~mm})$, as shown in Fig. 17 It is shorter than the ideal beam structure defined in the numerical study in order to accommodate laboratory space limitations. The structure is 


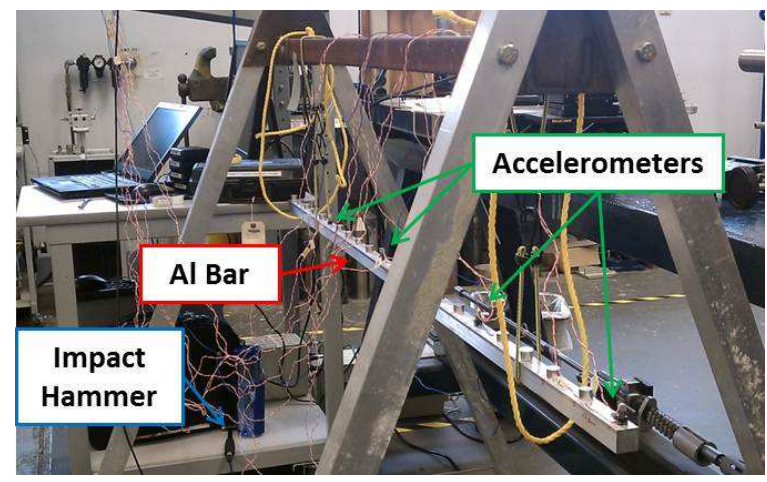

Figure 17: Photograph of the experimental setup.

range of 0.5 to $4000 \mathrm{~Hz}$, distributed evenly along its length. This number of accelerometers allows for good resolution in the slope information calculated from the acceleration data. These are triaxial accelerometers (axial, transverse and lateral directions), but only transverse response data is used.

The acceleration data is collected with a sampling frequency of $102.4 \mathrm{kHz}$ in order to capture the response with good temporal resolution. The data is downsampled by a factor of four in order to accommodate memory limitations when applying the force identification procedure. The structure is suspended from bungee cords in order to approximate free-free boundary conditions. A PCB 086D05 modal impact hammer is used to apply impulsive loads in the vertical direction. The applied force is measured by the force transducer in the impact hammer for comparison with the identified force information. For transducer signal conditioning and A/D signal conversion, a LMS SCADAS III acquisition system is used. The measured data is recorded by using LMS Test.Lab Software, Ver. 11A. The acceleration data sets are chosen from the accelerometers in the vicinity of the impact location. The acceleration data contains the response signal of the structure due to the impact and also the reflections due to the boundaries of the structure. The acceleration data is dispersive due to the dispersive nature of the structure.

The impact force identification method is applied to experimental response 


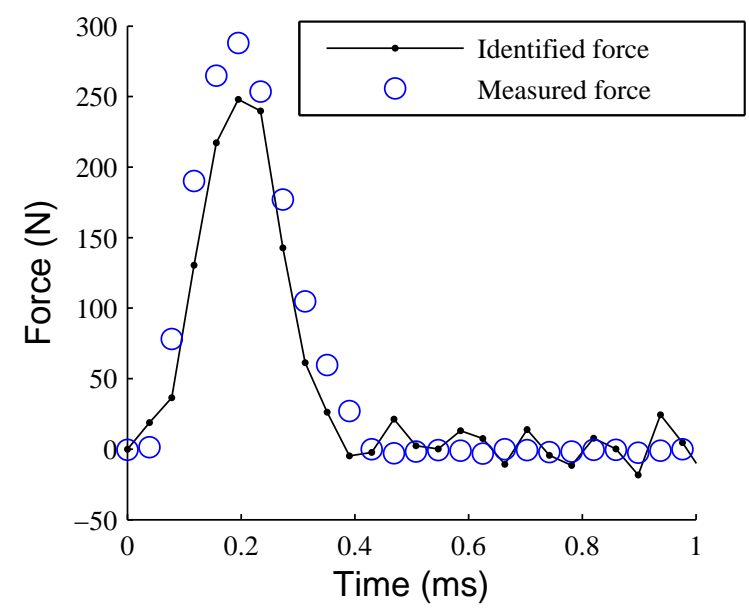

Figure 18: Force information calculated from the experimental data, force applied at node 11.

data collected for conditions when the impact location is collocated with an accelerometer and when the impact is applied between accelerometers. Representative results for these conditions are presented and discussed below.

As it was shown in the parametric study, the calculated impact force is more precise at cases where the impact occurs close to the middle of the structure. The first data set presented corresponds to an impact force being applied to the experimental system at the location of accelerometer number 11 near the middle of the structure. The force information obtained by applying the force identification method is presented in Fig. 18. Since the impact force was applied at the location of the accelerometer, the location of the impact is easily identified as the position of accelerometer 11. The identified force information agrees well with the measured force, successfully capturing the qualitative characteristics of the impulsive load. However, some discrepancies are observed. This error is believed to be introduced as a result of errors in the slope calculation due to signal noise and also due to the low frequency stop-band of the accelerometers which are used to perform modal analysis. The RMS error for this case is $34.60 \mathrm{~N}$ and an average value of $10.45 \mathrm{~N}$ exists during the remainder of the $1 \mathrm{~ms}$ time 


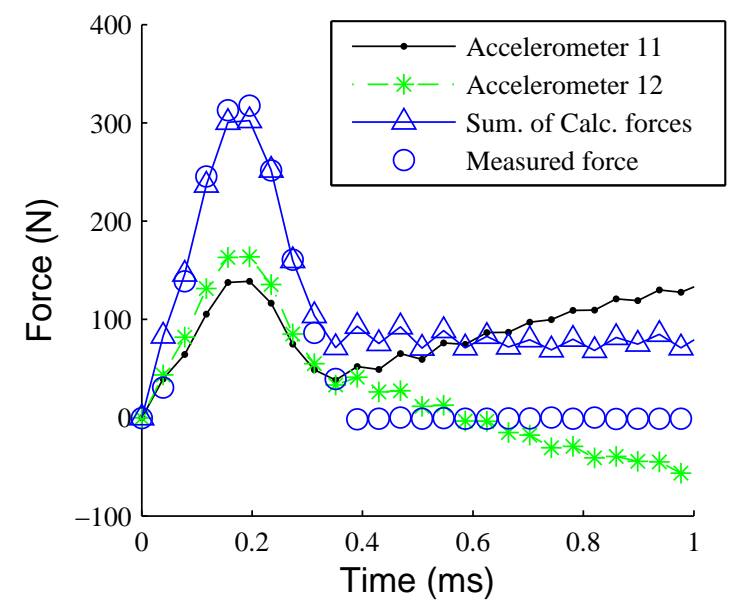

Figure 19: Force information calculated using experimental data for the force applied at $50 \%$ of the distance between the accelerometers from accelerometer 11 .

window presented in Fig. 18, In comparison with the ideal conditions, the length of the beam is shorter and noise is present in the acceleration data. While each of these differences contributes to the reduced accuracy of the calculated force information, the RMS error for the impulsive load is only $12 \%$ of the maximum force value.

The next two data sets presented were collected when the impact force was applied between accelerometers. The impact force identification method is applied to calculate the forces applied to the structure and the location identification method is used to determine where the impact force was applied. The first of these two data sets correspond to conditions where the impact force is applied at the center between accelerometers 11 and 12 . The impact force information obtained from the force identification method is presented in Fig. 19, The detected force values at nodes 11 and 12 exhibit similar properties since the impact was applied at equal distances from the two accelerometers. However, after the impulsive load, the identified force values increase or decrease linearly with time.

The summation of the identified forces at the accelerometers on either side of 


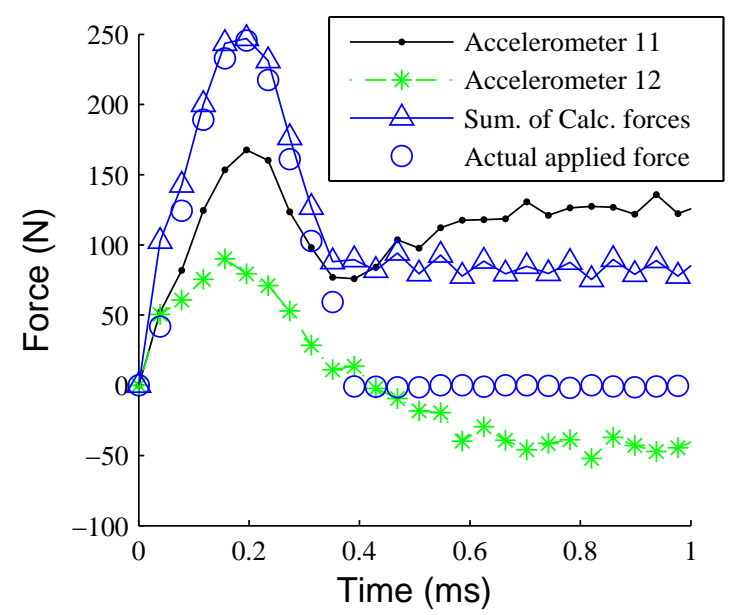

Figure 20: Force information calculated using experimental data for the force applied at $40 \%$ of the distance between the accelerometers from accelerometer 11. tion $(\Delta)$ agrees well with the measured force information (o) during the impact. After the impact, the calculated force exhibits a persistent offset from the measured force values. The RMS error for the summation of the two calculated forces is $39.20 \mathrm{~N}$ during the impact and averages $53.21 \mathrm{~N}$ during the remainder is believed to be due to the short length of the acceleration data set. Access to a longer set of the response data is expected to yield more accurate results. The bungee cords supporting the experimental system also apply a small but constant force to the structure which is not addressed in the model and may influence the results. By using the impulse ratio calculated for the force values at accelerometers 11 and 12, the impact location is calculated to be at a distance of $0.52 \mathrm{~L}$ from accelerometer 11 . This corresponds to a deviation of only $2 \%$ of the distance between the accelerometers which is less than $2 \mathrm{~mm}$.

The final data set correspond to conditions where the impact force is ap650 plied at a distance of $0.40 \mathrm{~L}$ from accelerometer 11 . The impact force information obtained from the force identification method is presented in Fig. 20. As 
the numerical study predicted, the calculated force values for the accelerometer nearest to the impact location are larger than the values for the other location. Again, the calculated force values at the positions of the two accelerometers gradually increase or decrease after the impact. The identified force information is obtained from the summation of the calculated forces and it agrees well with the measured force during the impact. The RMS error is $35.61 \mathrm{~N}$ during the impact and averages $83.78 \mathrm{~N}$ during the remainder of the $1 \mathrm{~ms}$ presented in Fig. 20. The impact force is identified to be at a distance of $0.37 \mathrm{~L}$ from accelerometer 11. This corresponds to a deviation of only $3 \%$ of the distance between the accelerometers which is less than $3 \mathrm{~mm}$ from the actual location of the applied impact force. Considering the fact that the impact was manually applied and the hammer tip is $6 \mathrm{~mm}$ wide, the errors in the results of the location identification method are acceptable.

The experimental verification illustrates a good agreement between the experiment and the simulation predictions. However, the force identification method of the experimental setup might experience errors due to the discrepancies in the material properties, loading conditions, mass of accelerometers and other different unknown factors. These errors can be reduced by conducting additional tests in order to calibrate the method.

\section{Concluding Remarks}

An impact force identification method using the spectral finite element method was introduced and studied with a beam structure. With this method, the impact force can be determined without precise information about the impact location. The procedure was studied with simulated response data for propagating mechanical waves and validated by using experimental data. In simulations, excellent agreement was observed for nominal conditions. Sources of error in the identified force information were investigated through a parametric study. The most influential parameter in the force calculation procedure is the length of 
structure length, moving the impact location toward the end of the structure, and increasing the impact duration. Also, the trends associated with varying the structure length, impact position, and impact duration were studied.

The proposed force identification method was applied to experimental data and was able to successfully identify the characteristics of the impact and provide identified impact force information with an RMS error of $34.60 \mathrm{~N}$ for an impact with a maximum force of $288 \mathrm{~N}$. In conjunction with the force identification method, a new technique for accurately determining the location of the impact was proposed. The location identification method utilizes the ratio of forces calculated at the positions of the neighboring accelerometers in order to identify the location of the impact. When applied to experimental data, the impact locations were identified within $5 \%$ of the distance between the accelerometers from the actual location, which corresponds to an error of less than 0.17 in $(0.4 \mathrm{~cm})$ on a $6 \mathrm{ft}(1.83 \mathrm{~m})$ structure.

695

An offset observed between the identified force and the measured force after the impulsive load has been identified to result from the use of response data of insufficient length. This characteristic will be further studied in future work in order to improve the understanding of the effect when the force is applied between sensors in order to more accurately identify the duration of the impulsive load. Future work will also explore the implementation of the force and location identification methods by using a wavelet based SFEM model as well as adapting these methods for nonlinear wave propagation data. The use of gyroscopic sensors will also be explored to provide more accurate rotational response data. The effect of uncertainties such as boundary conditions, mass of accelerometers, and the errors in the SFEM model will be studied by calibrating and conducting additional tests. While this work has demonstrated the application of the proposed methods to beam structures, its application to structures such as rods, plates and more complicated structures such as shells will also be explored in the future works. 
6] V. I. Bateman, T. G. Carne, D. L. Gregory, S. W. Attaway, H. R. Yoshimura, Force reconstruction for impact tests, Journal of Vibration and Acoustics 113 (2) (1991) 192-201.

[7] M. Mitra, S. Gopalakrishnan, Spectrally formulated wavelet finite element for wave propagation and impact force identification in connected 1-D waveguides, International Journal of Solids and Structures 42 (16) (2005) $4695-4721$. 
[8] J. F. Doyle, Further developments in determining the dynamic contact law, Experimental Mechanics 24 (4) (1984) 265-270.

[9] C. Chang, C. T. Sun, Determining transverse impact force on a composite laminate by signal deconvolution, Experimental Mechanics 29 (4) (1989) 414-419.

[10] V. Calhoun, X. Golay, G. Pearlson, Improved fMRI slice timing correction: interpolation errors and wrap around effects, in: Proceedings, ISMRM, 9th Annual Meeting, Denver, 2000, p. 810.

[11] S. Liu, Q. Wang, G. Liu, A versatile method of discrete convolution and FFT (DC-FFT) for contact analyses, Wear 243 (1) (2000) 101-111.

[12] Y. Keller, A. Averbuch, M. Israeli, Pseudopolar-based estimation of large translations, rotations, and scalings in images, Image Processing, IEEE Transactions on 14 (1) (2005) 12-22.

[13] G. V. Narayanan, D. E. Beskos, Use of dynamic influence coefficients in forced vibration problems with the aid of fast fourier transform, Computers \& Structures 9 (2) (1978) 145-150.

[14] J. F. Doyle, Wave Propagation in Structures: Spectral Analysis Using Fast Discrete Fourier Transforms, Springer, New York, NY, 1997.

[15] W. M. Ostachowicz, Damage detection of structures using spectral finite element method, Computers \& Structures 86 (3) (2008) 454-462.

[16] M. Krawczuk, Application of spectral beam finite element with a crack and iterative search technique for damage detection, Finite Elements in Analysis and Design 38 (6) (2002) 537-548.

760 [17] J. F. Doyle, T. N. Farris, A spectrally formulated finite element for wave propagation in 3-D frame structures, International Journal of Analytical and Experimental Modal Analysis 5 (1990) 223-237. 
[18] spectrally formulated finite element, European Journal of MechanicsA/Solids 41, (2013) 1-15 V. Ajith, S. Gopalakrishnan, Wave propagation in stiffened structures using

[19] J. F. Doyle, An experimental method for determining the location and time of initiation of an unknown dispersing pulse, Experimental Mechanics 27 (3) (1987) 229-233.

[20] C. Chen, F. G. Yuan, Impact source identification in finite isotropic plates using a time-reversal method: theoretical study, Smart Materials and Structures 19 (10) (2010) 105028.

[21] E. Wu, J. C. Yeh, C. S. Yen, Impact on composite laminated plates: an inverse method, International Journal of Impact Engineering 15 (4) (1994) $417-433$.

775 [22] L. Gaul, S. Hurlebaus, Identification of the impact location on a plate using wavelets, Mechanical Systems and Signal Processing 12 (6) (1998) 783-795.

[23] T. Kundu, S. Das, S. A. Martin, K. V. Jata, Locating point of impact in anisotropic fiber reinforced composite plates, Ultrasonics 48 (3) (2008) 193-201.

[24] J. H. Park, Y. H. Kim, Impact source localization on an elastic plate in a noisy environment, Measurement Science and Technology 17 (10) (2006) 2757.

[25] M. Meo, G. Zumpano, M. Piggott, G. Marengo, Impact identification on a sandwich plate from wave propagation responses, Composite Structures 71 (3) (2005) 302-306.

[26] D. Liang, S.-F. Yuan, M.-L. Liu, Distributed coordination algorithm for impact location of preciseness and real-time on composite structures, Measurement 46 (1) (2013) 527-536. 
[27] R. Seydel, F. K. Chang, Impact identification of stiffened composite panels: I. system development, Smart Materials and Structures 10 (2) (2001) 354.

[28] N. Hu, H. Fukunaga, S. Matsumoto, B. Yan, X. H. Peng, An efficient approach for identifying impact force using embedded piezoelectric sensors, International Journal of Impact Engineering 34 (7) (2007) 1258-1271.

[29] J. Park, S. Ha, F. K. Chang, Monitoring impact events using a systemidentification method, AIAA journal 47 (9) (2009) 2011-2021.

[30] Z. Boukria, P. Perrotin, A. Bennani, Experimental impact force location and identification using inverse problems: application for a circular plate, International Journal of Mechanics 5 (1) (2011) 48-55.

[31] K. Shin, H. Yang, S.-K. Lee, Y.-S. Lee, Group delay based location template matching method for the identification of the impact location on a plate, Journal of Sound and Vibration 332 (8) (2013) 2111-2117.

[32] P. Ghaderi, A. J. Dick, J. R. Foley, G. Falbo, Spectral domain force identification of impulsive loading in beam structures, in: IMAC XXX: A Conference and Exposition on Structural Dynamics, Jacksonville, Florida, 2012.

[33] P. Ghaderi, A. J. Dick, J. R. Foley, G. Falbo, High fidelity force location identification in beam structures, in: 83rd Shock and Vibration Symposium, New Orleans, LA, 2012.

[34] J. E. Akin, Finite Element Analysis with Error Estimators: An Introduction to the FEM and Adaptive Error Analysis for Engineering Students, Elsevier, Burlington, MA, 2005.

[35] W. Fladung, R. Rost, Application and correction of the exponential window for frequency response functions, Mechanical Systems and Signal Processing 11 (1) (1997) 23-36.

[36] A. V. Idesman, A new high-order accurate continuous galerkin method for 815 linear elastodynamics problems, Computational Mechanics 40 (2) (2007) 261-279. 
[37] , B. Y. Mao, S. L Xie, M. L, Xu, X. N, Zhang, G. H, Zhang, Simulated and experimental studies on identification of impact load with the transient statistical energy analysis method, Mechanical Systems and Signal Processing, 46 (2) (2014), 307-324.

[38] , R., Sun, G., Chen, H., He, B. Zhang, The impact force identification of composite stiffened panels under material uncertainty, Finite Elements in Analysis and Design, 81, 2014, 38-47.

[39] , S. Y., Khoo, Z. Ismail, K. K., Kong, Z., C, Ong, S., Noroozi, W., T., Chong, A., Rahman, Impact force identification with pseudo-inverse method on a lightweight structure for under-determined, even-determined and over-determined cases, International Journal of Impact Engineering, 63 (2014), 52-62. 